\title{
Ekbom syndrome episode associated to B12 Vitamin deficit
}

\section{שII \\ CENTRO \\ HOSPITALAR \\ PSIQUIATRIC
DE LISBOA}

\section{Introduction:}

Reports of patients with delusion of parasitosis (DP) were first seem in the late 19th century when Thibierge reported cases of "acrophobia". However, only in 1938 Ekbom's syndrome was first clinically described by Karl Axel Ekbom.

DP or Ekbom syndrome is a psychiatric condition in which the patients have a false and unshakeable belief that the body skin is infested by parasites. It can be idiopathic or secondary to organic or psychiatric diseases.

Typically is present the "matchbox sign", with the patient bringing proof of the infestation in small boxes. It can occur at any age, although the average is in the fifth decade, particularly among females. They normally are lonely persons, with no prior psychotic history.

This condition was classically treated with the antipsychotic pimozide, however now days are used atypical antipsychotics with better side-effect profile.

\section{Methods:}

Clinical case description and a non-systematic review through PubMed research about "delusion of parasitosis" and "Ekbom syndrome". A brief review of the theme was performed.

\section{The case:}

A 43-year-old Indian woman with a history of a depressive episode was taken to urgency department anguished, with delusional ideas of infestation associated with anxiety, starting 3 weeks before. Were also described depressive symptoms with 2 months of evolution. The cranial CT scan was normal and the laboratory studies revealed undetectable B12 vitamin.

She was living in Portugal for 15 years, residing in Lisbon with her husband and sons, without structured professional activity.

Stands out from her medical history a total hysterectomy with bilateral annexectomy. Was also being followed in dermatology for facial melasma. Familiar psychiatric history was absent, yet she described personal history of a depressive episode during her 2nd son's pregnancy, which has spontaneously remitted after birth. She denied any substance abuse or allergies.

Sensibly 2 months before hospitalization, she slowly starts with symptoms of abulia, adynamia, intermediate insomnia, and anorexia.
The case (continue):

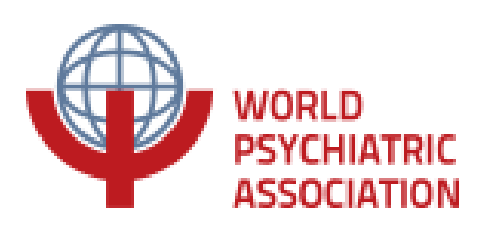

$19^{T H}$ WPA WORLD CONGRESS OF PSYCHIATRY LISBON, 21-24 AUGUST, 2019
One month after, she went to the urgency department with complaints of generalized pruritus. The common medical causes were excluded. About a week later, she gradually initiated with contamination ideas, that she associated with a hysterectomy procedure done 1 year before, and with possible transmission by her two friends, starting to avoid them. She also prevented contact with her sons because she feared to contaminate them.

3 weeks later, she was taken again to the urgency department, this time reporting pain and external genital pruritus. Was initially observed by a gynecologist and then referred to psychiatric observation. There she described tactile sensations like "water drops" in the body extremities, head, and genital area, that she interpreted as a result of microbes presence in the skin: "microbes are creeping through my body, coming out of my hands, head, and vagina, then entering in again...".

She presented a full conviction, not permeable to logical argumentation and were not present other types of delusions.

During one month span, the patient's condition slowly improved, with sertraline titrated to $200 \mathrm{mg} /$ day, olanzapine $15 \mathrm{mg} /$ day and $\mathrm{B} 12$ vitamin reposition, which resulted in the remission of the delusional ideas, abnormal tactile perceptions and depressive symptoms with a gradual insight development.

\section{Results and Conclusions:}

This case illustrates a middle-aged Indian woman with a history of depression, that initiates delusional infestation ideas during an ongoing depressive episode.

The laboratory studies revealed an undetectable B12 vitamin. The symptoms of this deficit are mainly hematological and neurological. The neurological symptoms consist of paraesthesia, loss of reflexes, lethargy, irritability, loss of concentration, depression, confusion and memory problems.

The patient was indeed going through a depressive episode when the infestation ideas started. They were described as a sensation of "water drops" in the body extremities, compatible with paresthesias.

We hypostatize that this vitamin deficit may have contributed to the genesis of the patient's delusional ideas. Thus, the contamination ideas might have probably been born from a wrong interpretation of the paraesthesias, that have gained delusional intensity, most probably secondary to the ongoing major depressive episode. 